\title{
REVISIÓN
}

\section{Utilización del ensayo del ácido 2-tiobarbitúrico (ATB) para evaluar el proceso autooxidativo en alimentos}

\author{
Por I. M. Vicario Romero, R. Guillén Sans y M. Guzmán Chozas*
}

Área de Nutrición y Bromatología. Departamento de Bioquímica, Bromatología y Toxicología. Facultad de Farmacia. Universidad de Sevilla. c/. Prof. García González, s/n. 41012 Sevilla.

\section{RESUMEN}

Utilización del ensayo del ácido 2-tiobarbitúrico (ATB) para evaluar el proceso autooxidativo en alimentos

En esta revisión científica se recogen diferentes aspectos de la aplicación del ensayo del ácido 2-tiobarbitúrico (ATB) a la medida del grado de autooxidación de alimentos lípidicos. Se consideran las diferentes formas de procesado del alimento, de inducción voluntaria a la autooxidación, de preparación de las muestras, técnicas empleadas y posibles interferencias.

Se contempla el estudio y aplicación del método a muestras de carne, pescados, huevos, leche y derivados lácteos, cereales, productos dietéticos infantiles, grasas animales y aceites vegetales comestibles. Se hace referencia a la posible correlación con otros índices de oxidación ya establecidos, como el índice de peróxidos y otros, así como también la posible correlación favorable entre los valores del índice o número de ATB y la evaluación sensorial del proceso autooxidativo (grado o estado de rancidez).

PALABRAS-CLAVE: Acido 2-tiobarbitúrico - Alimento Autoxidación - Rancidez - Revisión (artículo).

\section{SUMMARY}

Use of the 2-thiobarbituric acid (TBA) test for evaluation of the autoxidative process in foods.

In this overview several aspects concerning the application of 2-thiobarbituric acid (TBA) test for measuring oxidative rancidity in foods are described and discussed. Different factors like food processing, sample induced oxidation and preparation, applied methodologies and interferences, are considered. The application of the TBA test to some foods like meats, fish, eggs, milk and dairy products, cereals, infant-foods, animal fats and vegetable oils are described. Correlation between TBA index or TBA number and other oxidation indices (i.e. peroxide value) as well as sensory evaluation are also considered.

KEY-WORDS: Autoxidation - Food - Rancidity - Review (paper) - 2-Thiobarbituric acid.

\section{INTRODUCCIÓN}

El ensayo del ácido 2-tiobarbitúrico (ATB) tiene su origen en un trabajo publicado por Kohn y Liversedge
(1944) sobre los productos de oxidación de tejidos animales. Estos autores sugirieron que la sustancia reaccionante debería tratarse de un compuesto carbonílico. Bernheim et al. (1948) estudiaron la reacción entre el ATB y los productos de oxidación de determinadas sustancias lipídicas. De acuerdo con estas experiencias pioneras los productos de reacción coloreados, originados a partir de la oxidación de ácidos grasos insaturados, y su posterior reacción con ATB, se debían a la formación de los ácidos alquilidentiobarbitúricos.

Los primeros científicos que utilizaron esta reacción para detectar la autooxidación en alimentos, más concretamente en grasa de leche, fueron Patton y Kurtz (1951). Estos autores comprobaron que el espectro de absorción obtenido al hacer reaccionar la grasa de leche oxidada con ATB era similar al del pigmento rojo $\left(\lambda_{\operatorname{máx}}=530-532 \mathrm{~nm}\right)$ que se obtenía cuando reaccionaban directamente malondialdehído (MDA) y reactivo ATB. A partir de estas observaciones extrajeron la conclusión de que el MDA tenía una especial significación o protagonismo en la incidencia de la rancidez (término sensorial que se deriva del proceso de autooxidación de las grasas) de la grasa alimentaria, y en la oxidación de las membranas tisulares de los tejidos vivos, en general.

Si realizamos una revisión de la bibiliografía acumulada referente al ensayo del ATB, tres han sido las técnicas generalmente empleadas:

- La técnica de destilación, de Tarladgis et al., (1960), aplicada habitualmente a alimentos de origen animal.

- La de calentamiento a reflujo (Caldwell y Grogg, 1955; Cain et al., 1956; Pokorny y Dieffenbacher, 1989), sin etapa de separación previa.

- La de extracción simple, aplicada por primera vez a los aceites vegetales por Sidwell et al. (1954), y con modificaciones recientes en las que se introduce la extracción en fase sólida para su aplicación en carnes (Raharjo et al., 1993).

Mientras que en la técnica de extracción simple se extraen de la muestra las sustancias reaccionantes con ATB, empleando una fase acuosa en la que está disuelto el reactivo y posteriormente se calienta, en la 
técnica de calentamiento a reflujo la reacción se realiza directamente entre la muestra y el reactivo. En la técnica de destilación se hace una separación previa de las sustancias reactivas por volatilización a altas temperaturas. Existen adecuadas revisiones donde se comparan las diferentes técnicas en su aplicación a los aceites vegetales comestibles (Guillén-Sans y Guzmán-Chozas, 1993; Sans et al., 1993) o a carnes (Sorensen y Jorgensen, 1996).

Aunque, dada la complejidad del proceso, todavía no se ha llegado a esclarecer por completo el mecanismo de esta reacción, sí que se ha demostrado su utilidad, junto con otras técnicas de medida de la autooxidación, en la detección de los cambios oxidativo que se producen en los alimentos y en los sistemas biológicos por factores tales como el aire (oxígeno), el calor, la luz, las radiaciones ionizantes y la presencia de iniciadores de la reacción en cadena, tales como las trazas de elementos metálicos (cobre, hierro...). Una de las críticas frecuentes a esta reacción es su poca especificidad, ya que otros aldehídos presentes en el alimento también reaccionan con ATB dando un compuesto coloreado, que puede interferir en la evaluación total de la oxidación (Guillén-Sans y GuzmánChozas, 1995).

\section{CARNES}

El ensayo del ATB se ha aplicado a muestras de ganado bovino y de cerdo, y también en menor extensión, a muestras de pollo y de pavo. El procesado o preparación de la muestra ha sido muy diverso. Así, el ensayo del ATB ha sido practicado en carnes sometidas a diversos procesos de conservación: refrigeradas y/o congeladas (Tomás y Funes, 1987), carnes cocinadas (Tarladgis et al., 1960; Shaidi et al., 1987), carnes curadas (Zisper y Watts, 1962; Yun et al., 1987) o almacenadas durante distintos períodos de tiempo. Se han comparado los resultados obtenidos en carne cruda y sometida a diversos procesos de cocinado (asado, estofado, enlatado, cocinado lento y appertización o enlatado) (Huang y Greene, 1978). Por el frecuente uso de la misma, también se han hecho estudios del ensayo del ATB en carne picada (hamburgesas) (Greene y Cumuze, 1981; Willemont et al., 1987; Raharjo et al., 1991). En muestras refrigeradas $\left(4^{\circ} \mathrm{C}\right)$ y congeladas $\left(-20^{\circ} \mathrm{C}\right)$ se daban valores más bajos del número de ATB que los que se daban en carne cocida.

La técnica habitualmente empleada ha sido la destilación (Tarladgis et al., 1960), que en ocasiones se ha comparado con la técnica de calentamiento a reflujo, convencional después de desproteinizar con ácido tricloroacético (ATA) (Tomás y Funes, 1987). Al objeto de reducir el tiempo de duración de la técnica de destilación, a veces ardua, en carne de bovino picada se empleó el calentamiento en autoclave (Ramírez y
Spillman, 1987). Asimismo se ha usado la técnica de extracción simple modificada, empleando como extractante el ácido perclórico. Se pudo concluir que la técnica de extracción era más rápida, fácil de llevar a cabo y tan exacta como lo pudiera ser la de destilación, en el control de carne de aves (Salih et al., 1987). Una mejora de la técnica de extracción simple, usando ATA, permite medir a baja temperatura $\left(4^{\circ} \mathrm{C}\right)$ y eliminar las sustancias que interfieren mediante columnas en fase sólida (C 18) (Raharjo et al., 1991).

Como longitud de onda experimental (de medida), se escogió la que correspondía al máximo de absorción del pigmento rojo (530-532 nm). Se han ofrecido fórmulas correctivas para evitar las interferencias (si las hubiera) de los pigmentos amarillo $\left(\lambda_{\text {máx }}=450 \mathrm{~nm}\right)$ y/o anaranjado ( $\lambda_{\text {máx }}=497 \mathrm{~nm}$ ) (Yu y Sinnhuber, 1962). Para calcular el número de ATB se ha hecho uso de sustancias modelo y del valor de absortividad molar del compuesto coloreado (Witas, 1972). A veces, mediante una aproximación matemática, se elimina la necesidad de usar un patrón externo (Izumimoto et al., 1990).

Diversos factores pueden afectar los resultados del ensayo. La adición de antioxidantes, como galato de propilo y AEDT ocasionaban valores más bajos del índice de ATB (Rhee., 1978). Iniciadores del proceso autooxidativo, como hematina (Keskinel et al., 1964), sales de Fe (III) o tert-butilhidroperóxidos (Kosugi et al., 1991), incrementaban este índice de ATB. El tipo de especie animal y alimentación (harina de pescado), clase de músculo o profundidad desde la piel de la toma de muestra (Arefjord, 1960), interferencias frecuentes (carbohidratos) y el tamaño y grado de división de la muestra (Turner et al., 1954), ocasionaban efectos diversos sobre los resultados. Dietas ricas en hierro incrementaban el valor de las sustancias que reaccionan con ATB (TBARS) en músculo esquelético de cerdo (Miller et al., 1994). La presencia de aditivos, como alginatos, aumentaba los índices del ensayo (Raharjo et al., 1989), en cambio la presencia de nitritos prevenía la rotura oxidativa de los lípidos durante el almacenamiento (Willemont, 1987). En carne de ternera a la que se había suministrado vitamina $E$ ofrecía índices de ATB más bajos (Shortland, 1976).

Se ha correlacionado el número de ATB con otros parámetros indicativos de la oxidación lipídica como el índice de peróxido (Vittuci et al., 1986) o el índice de yodo (Arefjord, 1960). En carne de bovino cruda los contenidos en hexanal y $2-3$, octanodiona mostraban un grado de correlación significativamente altos con los resultados obtenidos en el ensayo del ATB; la carne estaba cruda, recién cocida, almacenada y recocinada (St. Angelo et al., 1987). Después de aplicar la técnica de destilación, se encontraron coeficientes de correlación bajos, aunque significativos, entre el número de ATB y la puntuación sensorial, en carne de bovino picada (Greene y Cumuze, 1981). La extracción en fase sólida supone una mejora de la técnica de extracción simple, aumenta la especificidad y ofrece 
una buena correlación con la evaluación sensorial en carne de ternera cruda y cocinada (Raharjo et al., 1993).

\section{PESCADOS}

Los estudios se han centrado fundamentalmente en especies de origen marino: merluza y bacalao (Crawford et al., 1979), sardina (Asahara, 1987; Rodríguez et al., 1991), arenque (Snauwarert et al., 1977), atún (Sinnhuber y Yu, 1958), caballa (Vyncke, 1975), jurel (López Capont y Barcia, 1978) salmonete (Lee y Toledo, 1977), tiburón (Kakiuchi et al., 1989) y ostras (Schwartz y Watts, 1957). Aunque también existen un número estimable de referencias que se refieren a la carpa (Mai y Kinsella, 1979; Iredale y York, 1977) y al salmón (Vittucci et al., 1986). También se ha aplicado el método en harina, aceite y salsa de pescado.

La preparación de la muestra varía según los casos. Se ha controlado pescado fresco, congelado, refrigerado, almacenado varios meses, seco y salado, en filetes, rodajas, desmenuzado, picado, sin la piel, almacenado a una humedad relativa del $30-50 \%$, envasado a vacío o irradiado, cocinado o sometido a fritura. La rancidez del pescado y los métodos a través de los cuales puede detectarse, entre ellos el método del ATB, ha sido revisada (Careche y Jiménez-Colmenero, 1988). Como técnicas, se han empleado la destilación original (Rhee, 1978), la extracción directa utilizando ácido tricloroacético (Vyncke, 1978) y un procedimiento indirecto que suponía la extracción previa de los lípidos desde la muestra (Bratkoswska y Zwierzykowski, 1988); asimismo se ha utilizado el extractor Bligh y Dyer, que separa el MDA libre de MDA lábil, aconsejado para las muestras con muy bajo contenido graso.

Los resultados que se obtuvieron con la técnica de destilación original fueron distintos en muestras de pescado, que en muestras de animales terrestres (bovino, cerdo y pollo), lo que parece lógico debido a la diferente composición en ácidos grasos insaturados. Si se trata de muestras de peces, aún se hace más necesario el empleo de un sistema antioxidantesinérgico al aplicar la técnica de destilación (Rhee, 1978). La adición de la mezcla de tripolifosfato AEDT a pescado en salsa, aunque suponía un descenso en las cifras del número de ATB, no conseguía inhibir el olor a rancio. El uso de BHT era más efectivo para reprimir el ascenso del número de ATB; pero tampoco resultaba eficaz para suprimir el desagradable efecto sensorial. Solamente mediante el empleo de una mezcla ascorbato-nitrito se conseguía detener la apreciación del desarrollo oxidativo, tanto por las cifras que se obtenían del número de ATB, como en el aspecto sensorial (Satomi et al., 1988).

Hemoglobina, o mioglobina, y Fe (II), aumentaban las cifras del número de ATB en aceite de pescado, empleando la técnica de destilación. La adición de AEDT enmascaraba el Fe (II), mientras que la adición de BHA prevenía el efecto adverso de los pigmentos de la sangre (Shibata y Kinumaki, 1979 a, b). Otros factores que también pueden influenciar los resultados son el tipo de especie animal (Crawford et al., 1979), o el tipo de músculo (rojo o blanco) que se considera (Lee y Toledo, 1977). Una interferencia importante es la presencia de formaldehído $(\mathrm{HCHO})$, sustancia que se origina en el pescado. El formaldehído ocasiona una turbidez o precipitado blanco, cuando reacciona con el reactivo $A T B$, habiéndose comprobado que una concentración superior a $25 \mu \mathrm{g} / \mathrm{mL}$ interfiere claramente (Almandós et al., 1986). Para evitar un proceso de separación previa (filtración, centrifugación) se podría soslayar la interferencia empleando un exceso de reactivo; más concretamente, que la ratio concentración de ATB/concentración de MDA $\geq 1000$ (Careche y Tejada, 1988). El número de ATB se ha correlacionado satisfactoriamente con el contenido en la muestra de pescado de sustancias reductoras volátiles y con el índice de peróxido. Parece darse una buena correlación entre el ensayo del ATB y el método de HPLC con detector fotométrico (532 nm) (Kakiuchi et al., 1989).

\section{HUEVOS}

Los estudios se han centrado en yema de huevo almacenada varios días a $23^{\circ} \mathrm{C}$, empleando la técnica de destilación. Los resultados se ven afectados por factores tales como la presencia, con efecto pro-oxidante, tanto del hierro, naturalmente presente en la yema, como del cloruro de guanidina, presencia de antioxidantes, AEDT (secuestrante) (Pike y Peng, 1988a), y la influencia del $\mathrm{pH}$, puesto que a valores bajos del $\mathrm{pH}$ se incrementa la prooxidación (Pike y Peng, 1988b).

\section{LECHE Y DERIVADOS LÁCTEOS}

Leche (entera y en polvo) y derivados lácteos, como la caseína (Witas, 1972), fueron los primeros productos alimentarios a los que se le aplicó el método del ATB (Dunkley y Jennings, 1951), así como mantequilla y queso, comprobándose que este ensayo mide el grado de oxidación por debajo del nivel de sensibilidad alcanzable sensorialmente (Biggs y Bryant, 1953). Las muestras se almacenaban a temperatura ambiente, refrigeradas y en salmuera, así como pasteurizadas. La técnica usualmente empleada es indirecta, mediante extracción previa de la fracción grasa.

La reacción se ve afectada por la interferencia de carbohidratos (lactosa) (Biggs y Bryant, 1953), y el tipo de disolvente usado en la extracción (Pearson, 1974). 
En muestras de mantequilla almacenada interfería la presencia de especias (comino, tomillo) en el producto original (Farag et al., 1988). En queso mantenido en salmuera se comprobó que tanto la refrigeración como la pasteurización reducían sensiblemente la autoxidación (Hamed et al., 1987). El índice de ATB en leches y derivados lácteos se han comparado con otros índices como el de Kreis, el índice de yodo, índice de acidez y peróxidos. Se comprobó (Patton y Kurtz, 1951) que en lácteos el ensayo del ATB era más sensible que los tradicionales ensayos del índice de yodo y del índice de Kreis, y presenta una correlación adecuada con las pruebas sensoriales (Dunkley, 1951).

\section{CEREALES, PRODUCTOS DE REPOSTERÍA Y PRODUCTOS DIETÉTICOS INFANTILES}

El ensayo del ATB se aplicó por primera vez en productos de panadería a mediados de la década de los cincuenta (Caldwell y Grogg, 1955). En productos procesados, tales como pasteles y salsas, se determinó el número de ATB, haciendo uso de la absortividad molar del pigmento rojo (Witas, 1972). En productos de pastelería elaborados con margarina y edulcorantes (sacarosa y maltitol), se comprobó mediante el ensayo del ATB que se manifestaba un mayor efecto prooxidante cuando en la composición el edulcorante usado era la sacarosa (Wada et al., 1978). Una modificación en el ensayo del ATB, en la que se calcula la absorbancia de los aductos ATB-MDA y ATB-sacarosa, permite corregir la interferencia debida a los azúcares (Du y Bramlage, 1992). Varios productos dietéticos infantiles se almacenaron en condiciones ordinarias durante dos años; periódicamente se investigaban distintos índices para evaluar el progreso de la oxidación, entre ellos la prueba del ATB, que fue la que mejor coeficiente de correlación presentaba con las pruebas sensoriales (Karadzhov, 1973).

\section{GRASAS ANIMALES}

El método se ha aplicado a sebo y manteca de cerdo sometidos a tratamiento térmico, que iba desde $120^{\circ} \mathrm{C}$ a $240^{\circ} \mathrm{C}$ (Kalac et al., 1988). Tradicionalmente se ha empleado la técnica de extracción simple (Sidwell et al., 1954; Vargas y Gutiérrez, 1956). Pokorny et al., (1985) desarrollaron una técnica adaptable a los primeros estadios de oxidación de la manteca de cerdo. Se han hecho otras pruebas con antioxidantes y sinérgicos, y también con cațalizadores clásicos del proceso autooxidativo (hierro, cobre e hidroperóxidos preexistentes en la muestra) (Kosugi et al., 1991). En manteca de cerdo existía una buena correlación entre las pruebas sensoriales y el número de ATB (Jacobson et al., 1964). Maleki (1974) llevó a cabo una validación del método de grasas y aceites.

\section{ACEITES VEGETALES COMESTIBLES}

El ensayo del ATB se ha aplicado a diversos aceites: aceite de oliva virgen y refinado, aceites de soja, aceites de girasol, sésamo, algodón, maíz, colza, cártamo y cacahuete. La introducción voluntaria y en un tiempo breve de la autooxidación en la muestra incluyó diversos procedimientos: hidrogenación parcial, conservación a la luz difusa, método del oxígeno activo (A.O.M.) (Sidwell et al., 1954; Gutiérrez y Vargas, 1957), almacenamiento en envases cerrados y abiertos durante varios meses (Solinas y Rossetti, 1977), envejecimiento de los aceites a $37^{\circ} \mathrm{C}$ y $60^{\circ} \mathrm{C}$ (Jacobson et al., 1964), la adición de antioxidantes y sinérgicos con posterior calentamiento entre $38^{\circ} \mathrm{C}-75^{\circ} \mathrm{C}$ (Fioriti et al, 1974), la termooxidación en estufa a distintas temperaturas (Arya y Nirmala, 1971; Kalac et al., 1988; Guillén-Sans et al., 1988; Guillén-Sans et al., 1991).

Las diferentes técnicas utilizadas para medir la autooxidación lipídica en aceites vegetales han sido revisadas recientemente (Guillén-Sans y Guzmán Chozas, 1993). Se ha empleado la técnica de extracción simple original, midiendo las absorbancias a 452 $\mathrm{nm}$ y $530 \mathrm{~nm}$. Reduciendo la temperatura de calentamiento, se consigue estabilizar el pigmento amarillo $(450 \mathrm{~nm})$ y se obtiene una mejor correlación con las pruebas sensoriales (flavor) que en la técnica original (Marcuse y Pokorny, 1994). En tiempos se utilizó un sistema cerrado y temperaturas de ebullición $\left(100^{\circ} \mathrm{C}\right)$ para llevar a efecto la reacción (Taüfel y Zimmerman, 1960). Utilizando sustancias modelo, y haciendo uso de la absortividad molar, se determinó el número de ATB a partir de los valores de absorbancia a $532 \mathrm{~nm}$ (Witas, 1972). Pero la técnica más adecuada ha demostrado ser la extracción simple, modificada y optimizada en aceites de oliva, aplicable con igual eficacia a los aceites de semilla (Vicario et al., 1988, 1990). Otras técnicas dispersas se han empleado. Papel reactivo de ATB, que contenía ácido cítrico y tiosulfato sódico, podría ser válida como prueba semicuantitativa (Asakawa y Matsushita, 1975).

En aceite de soja termooxidado se comprobó la existencia de correlación entre el incremento de MDA y el descenso de tocoferoles. Tanto MDA (malondialdehído) como las TBARS (sustancias que reaccionan con ATB) ascendían durante el proceso oxidativo (oxidación a $170^{\circ} \mathrm{C}$ ) en presencia de tocoferol (Kishida et al., 1991). Entre los valores TBARS y el consumo de oxígeno también se da una buena correlación, creciendo ambos linealmente hasta ciertos valores de consumo de oxígeno en los que los valores TBARS se estabilizan e incluso descienden (Kishida et al., 1993). En determinadas condiciones ni el índice de peróxidos ni el índice de Kreis eran pruebas adecuadas para determinar el período de inducción, pero sí se pudo lograr a través del ensayo del ATB (Gutiérrez y Vargas, 1960; Arya y Nirmala, 1971). El aceite de algodón, utilizado como muestra-sustrato, sirivió para un estudio 
comparativo del método del ATB frente a otros métodos y técnicas, tales como la quimioluminiscencia, HPLC, o la resonancia de spin electrónico (Yang et al., 1991). Entre los factores que afectan o pueden afectar, los resultados del ensayo se podría citar el tipo de aceite vegetal, su forma de envasado y el almacenamiento de las muestras (debido principalmente a la inestabilidad del MDA), junto con el tipo de técnica aplicado (Guillén-Sans et al., 1993), la elección adecuada de la longitud de onda de medida (532 nm, 452 $\mathrm{nm}$ ) y la posible presencia de sustancias que rebajan la oxidación (gliadina, albúmina) (Taguchi et al., 1988).

\section{BIBLIOGRAFÍA}

Almandós, M. E., Giannini, D. H., Ciarlo, A. S., Boeri, R. L. (1986). - «Formaldehyde as an interference of the 2thiobarbituric acid test".- J. Sci. Food Agric. 37, 54-58.

Arefjord, M. (1960). - «Content of thiobarbituric acid (TBA) and iodine number in different depths of fats".- Nord. Jordbrugforskn 42, 111-114.

Arya, S. S. y Nirmala, N. (1971). - «Determination of free malonaldehyde in vegetable oils".- J. Sic. Technol. (India) 8, 177-180.

Asahara, M. (1987). - «Antioxidant effects of natural tocopherol mixture, calcium phytate and inositol on marinated sardine».- Nippon Suisan Gakkaishi, 53, (9), 1617-1621.

Asakawa, T. y Matsushita, S. (1975). - «Test manufactured of test paper for detection of oxidized lipids».- Eiyo To Shokuryo, 28 (7), 403-405.

Bernheim, F., Bernheim, M. L. C. y Wilbur, K. M. (1948). -«The reaction between thiobarbituric acid and the oxidation products of certain lipids».- J. Biol. Chem. 174, 257-264.

Biggs, D. A. y Bryant, L. R. (1953). - «The thiobarbituric acid test for butterfat oxidation".- Can J. Technol. 31, 138-145.

Bratkowska, I. y Zwierzykowski, W. (1988). - "Comparison of methods for studying the degree of fish lipid oxidation".Izv. Vyssh. Uchebn. Zavel Pisch. Tekhnol. 5, 73-76.

Cain, R. F., Bubl, E. C., Anderson, A. W. (1956). - «The effect of intermitent radiations and concomitant increase in temperature during radiation on the acceptability of goung beef».- Food Technol. 10, 537-540.

Caldwell, E. F. y Grogg, B. (1955). - «Application of the thiobarbituric acid test to cereal and baked products».Food Technol. 9, 185-186.

Careche, M. y Jiménez Colmenero, F. (1988). - «Oxidación de lípidos en pescado: Procedimientos de determinación».Grasas y Aceites 39, 387-396.

Careche, M. y Tejada, M. (1988). - «Interference of formaldehyde in the 2-thiobarbituric acid test for rancidity".J. Sci. Food Agric. 43, 49-57.

Crawford, D. L., Law, D. K., Babbit, J. K. y McGill, L. A. (1979). - «Comparative stability and desirability of frozen Pacific hake fillet an minced flesh blocks». - J. Food Sci. 44, 363367.

Du, Z. Y., Bramlage, W. J. (1992). -«Modified thiobarbituric acid assay for measuring lipid oxidation in sugar-rich plant tissue extracts".- J. Agric. Food Chem. 40, 1566-1570.

Dunkley, W. L. (1951). - «Evaluation of the thiobarbituric acid as a measure of oxidized flavor in milk».- Food Technol. $5,342-346$

Dunkley, W. L., Jennings, W, G. (1951). - «A procedure for application of the thiobarbituric acid test to milk». - J. Dairy Sci. 34, 1064-1069.
Farag, R. S., Ali, M. N. y Taha, S. H. (1988). -«Use of some essential oils as natural preservatives for butter».-Grasas y Aceites 39, 28-31.

Fioriti, J. A., Kanuc, M. J. y Sims, R. J. (1974). -«Chemical and organoleptic properties of oxidized fats».- J. Am. Oil Chem. Soc. 51, 219-223.

Green B. E. y Cumuze, T. H. (1981). -«Relashionship between TBA number and inexperienced panelists' assessment of oxidized flavor in cooked beef».- J. Food Sci. 47, 52-58.

Guillén-Sans, R. y Guzmán-Chozas, M. (1993). -"Oxidative alterations of edible vegetable fats and oils measured by the thiobarbituric acid assay: applied methodologies».Rev. Franc. Corps Grass 40, 49-52.

Guillén-Sans, R. y Guzmán-Chozas, M. (1995). -«Aldehydes in food and its relation with the TBA test for rancidity". Fat Sci. Technol. 97, 285-286.

Guillén-Sans, R., Vicario I. y Guzmán-Chozas, M. (1988). -«El ensayo del ácido 2-tiobarbitúrico (ATB) en aceites vegetales comestibles".- Alimentaria. 193, 57-60.

Guillén-Sans, R., Yépez, F., Heredia, F. y Guzmán-Chozas, M. (1993). «Chromatic parameters and oxidation indices for edible vegetable oils submitted to thermal oxidation". -J. Sci. Food Agic. 54, 619-633.

Guillén-Sans, R., Vicario I. y Guzmán-Chozas, M. (1993) - CComparison of various procedures employed in the TBA test of spanish edible vegetable oils».- Fat. Sci. Technol. 95, 225-227.

Gutiérrez, R. y Vargas, A. (1957). -«Estudios sobre el enranciamiento del aceite de oliva. IX. La prueba del ácido tiobarbitúrico (TBA)».- Grasas y Aceites 8, 73-75.

Gutiérrez, R., Vargas, A. (1960). -«Estudios sobre el enranciamiento del aceite de oliva XI. Comparación entre las diferentes pruebas para la determinación del grado de rancidez".- Grasas y Aceites 11, 67-70.

Hamed, A. I., Farag, S. I. y Abou-Zeid, N. A. (1987). -«Studies on the effects of some factors on the oxidation of Domiati cheese fat».- Egypt. J. Dairy Sci. 15, 209-219.

Huang, W. H. y Greene, B. E. (1978). - Effect of cooking method on TBA number of stored beef».- J. Food Sci. 43, 1201-1203.

Irelade, D. G. y York, R. K. (1977). - «Effects of chemical additives on extending the shelf-life of frozen minced sucker flesh».- J. Fish Res. Board Can. 34, 420-425.

Izumimoto, M., Kataoka, K. y Miyamoto, T. (1990). -«Mathematical approach for determining TBARS in meat by extraction and distillation methods».- Agric. Biol. Chem. 54, 1311-1313.

Jacobson, G. A., Kirkpatrick, J. A. y Goff, A. E. J. (1964). -«A study on the applicability of a modified thiobarbituric acid test to flavor evaluation of fats and oils».- J. Am. Oil Chem. Soc. 41, 124-128.

Kakiuchi, Y., Yamauchi, A., Hama, S. y Yamazaki, H. (1989). - «Analysis of reaction products in the water extractionTBA method for fat deterioration testing".- Eisei Kagaku 35, 291-296

Kalac, J., Uhnak, J. y Rupcikova, P. (1988). -«The evaluation of changes in edible fats after thermal processing in a model test and in experiment".- Cesk. Hyg. 33, 316-322.

Karadzhov, I. (1973). - «Changes in fat quality properties of pureed infants foods during storage».- Nauch. Tr. Nachnoizsled Inst. Konserv. Prom. Plovdiv 10, 47-54.

Keskinel, A., Ayres, J. C. y Snyder, H. E. (1964). - «Determination of oxidative changes in raw meats by the 2-thiobarbituric acid method".- Food Technol. 18, 101-104.

Kishida, E., Kamura, A., Tokumaru, S., Oribe, M., Iguchi, H. y Kojo, S. (1993). - «Re-evaluation of malondialdehide and thiobarbituric acid reactive substances as indices of autoxidation based on oxygen consumption».- J. Agric. Food Chem. 41, 1-4.

Kishida, E., Oribe, M. y Kojo, S. (1991). «Relationship among malonaldehyde, TBA-reactive substances and tocopherols 
in the oxidation of soybean oil».- Nippon Kasei Gakkaishi 42, 219-222.

Kohn, H. I. y Liversedge, M. (1944). - «On a new aerobic metabolite whose production by brain is inhibited by apomorphine, ergotamine, epinephrine, and menadiona".J. Pharmacol. 82, 292-300.

Kosugi, H.; Kojima, T. y Kikugawa, K. (1991). - «Characteristic of the thiobarbituric acid reactivity of oxidized fats and oils". - J. Am. Oil Chem. Soc. 68, 51-55.

Lee, C. M. y Toledo, R. T. (1977). - «Degradation of fish muscle during mechanical deboning and storage with emphasis on lipid oxidation".- J. Food Sci. 42, 1646-1649.

López Capón, F. y Barcia, A. (1978). -«Talla, humedad, grasa y rancidez como índices de calidad del jurel (Trachurus trachurus, L.) seco-salado".- Rev. Agroquím. Tecnol. Aliment. 18, 505-511.

Mai, J. y Kinsella, J. E. (1979). - «Changes in lipid composition of cooked minced carp (Cyprinus carpio) during frozen storage". - J. Food Sci. 44, 1619-1624.

Maleki, M. (1974). - «Validity of the 2-thiobarbituric acid test for the quantitative determination of rancidity in fats and oils".- Fette, Seifen, Anstrichm. 76, 181-183.

Marcuse, R. y Pokorny, J. (1994). - «Higher correlation with sensory evaluation of oxidative rancidity by modified TBA test». - Fat Sci. Technol. 96, 185-187.

Miller, D. K., Gómez-Basauri, J. V., Smith, V. L., Kanner, J. y Miller, D. D. (1994). - «Dietary iron in swine rations affects nonheme iron and TBARS in pork squeletal muscles".- J. Food Sci. 59, 747-750.

Patton, S. y Kurtz, G. W. (1951). -«2-thiobarbituric acid as a reagent for detecting milk fat oxidation".- J. Dairy Sci. 34, 669-674.

Pearson, D. (1974). - «Assessment of rancidity values of oils on a common chloroform extract with special reference to TBA values".- J. Assoc. Public Anal. 13, 39-40.

Pike, O. A. y Peng, I. C. (1988a). - «Effect of protein disruption by denaturation and hydrolisis on egg yolk lipid oxidation».Food Sci. 53, 428-431.

Pike, O. A., Peng, I. C. (1988b). - «Influence of pH on egg yolk lipid oxidation».- J. Food Sci. 53, 1245-1246.

Pokorny, J. y Dieffenbacher, A. (1989). - «Determination of 2thiobarbituric acid value: direct method. Results of a collaborative study and the standarized method".- Pure Appl. Chem. 61, 1165-1170.

Pokorny. J., Valentová, H. y Davidek, J. (1985). -«Modified determination of 2-thiobarbituric acid value in fats and oils". - Nahrung-Food 29, 31-38.

Raharjo, S., Sofos, J. N., Maga, J. A. y Schmidt, G. R. (1989). - «Influence of meat restructuring systems on lipid oxidation in beef".- Lebesm. Wiss. Technol. 22, 199-203.

Raharjo, S., Sofos, J. N., Schmidt, G. R., y Maga, J. A. (1991). - «A specific thiobarbituric acid method for measuring lipid peroxidation in ground beef ".- en Proceedings Anual Reciprocal Meat Conference of the American Meat Science Association».-44, p. 204.

Raharjo, S., Sofos, J. N., y Schmidt, G. R., (1993) -«Solidphase acid extraction improves thiobarbituric acid method to determine lipid oxidation".- J. Food Sci. 58, 921-924, 932.

Ramírez, M. y Spillman, D. M. (1987). -«A rapid method for the determination of thiobarbituric acid reactive substances".- J. Food Sci. 52, 500-502.

Rhee, K. S. (1978). - «Minimization of further lipid peroxidation in the distillation 2-thiobarbituric acid test of fish and meat".. J. Food Sci. 43, 1776-1781.

Rodríguez, C. J., Velasco, F., Besteiro, I., Rodríguez, S., Quintana, R. y Pascual, M. C. (1991). - Evaluación sensorial y química de la sardina (Sardina pichardus, Walb.) almacenada en hielo y cámara fría".- Alimentaria 219, 8792.

Salih, A. M. Smith, D. M. Price, J. F. y Dawson, L. E. (1987). - «Modified extraction 2-thiobarbituric acid method for measuring lipid oxidation in poultry».- Poult. Sci. 66, 14831488.

Sans, R. G., Romero, I. M. y Chozas, M. G. (1993). - "Comparison of Various Procedures Employed in the TBA est of Spanish Edible vegetable Oils". - Fat Sci. Technol. 6, 225-227.

Satomi, K., Sasaki, A. y Yokoyama, M. (1988). - «Lipid oxidation of fish sausage and its inhibition-III. Effect of chelating agents and antioxidants on the lipid oxidation of fish sausage».- Nippon Suisan Gakkishi 54, 107-112.

Schwartz, M. G. y Watts, B. M. (1957). -«Application of the thiobarbituric acid test as an quantitative measure of deterioration in cooked oysters".- Food Res. 22, 76-82.

Shaidi, F., Yun, J., Rubin, L. J., Wood, D. F. (1987). -«The hexanal content as an indicator of oxidative stability and flavor acceptability in cooked ground pork».- Can Inst. Food Sci. Technol. J. 20, 104-106.

Shibata, N. y Kinumaki, T. (1979a). - «An improvement of TBA procedure as the measure of the oxidative deterioration ocurring in fish oils. I. Distillation procedure».- Nippon Suisan Gakkaishi 45, 499-503.

Shibata, N. y Kinumaki, T. (1979b). - «An improvement of TBA procedure as the measure of the oxidative deterioration ocurring in fish oils. II. Intact sample procedure».- Nippon Suisan Gakkaishi 45, 505-509.

Shortland, F. B. (1976). - -Deteriorative changes in frozen meats".-Meat Ind. Res. Inst. N. Z. (Rep) M.I.R.I.N.Z. 519, 83-89.

Sidwell, C. G., Salwin, H., Benca, M. y Mitchell, J. H. J. (1954). - «The use of thiobarbituric acid as a measure of fat oxidation".- J. Am. Oil Chem. Soc. 31, 603-606.

Sinnhuber, R. y Yu, T. C. (1958). - «2-Thiobarbituric acid method for the measurement of rancidity on fishery products. II. The quantitative determination of malonaldehyde».- Food Technol. 12, 9-12.

Snauwaert, F., Tobback, P., Maes, E. y Thyseen, J. (1977). - «Radiation induced lipid oxidation in fish».- Z. Lebensm. Unters. Forsch. 164, 28-30.

Solinas, M. y Rossetti, D. (1977). - «Some analytical indexes on the evaluation of the storage stability of an edible oil».Bol. Chim. Unione Ital. Lab. Prov. 3, 65-67.

Sorensen, G. y Jorgensen, S. S. (1996). - «A critical examination of some experimental variables in the 2-Thiobarbituric acids (TBA) test for lipid oxidation in meat products". $-Z$. Lebensm. Unters.-Forsch 202, 205-210.

St. Angelo A. J. Vercelloti, J. R. y Legendre, M. G. (1987). - «Chemical and instrumental analyses of warmed-over flavor in beef».- J. Food Sci. 52, 1163-1168.

Taguchi, K., Iwami, K., Kawabata, M. y Ibuki, F. (1988). - «Antioxidant effect of wheat gliadin and hen's egg white in powder model systems: protection against oxidative deterioration of safflower oil and sardine oil».- Agric. Biol. Chem. 52, 539-545.

Tarladgis, B. G., Watts, B. M., Younathan, M. T. y Dugan, L. R. Jr. (1960). -A distillation method for the quantitative determination of malonaldehyde in rancid foods. J. Am. Oil Chem. Soc. 37, 44-48.

Taüfel, K. y Zimmerman, R. (1960). -«Carbonyl formation in autoxidized fats".- Ernahrungsforschung 5, 104-109.

Tomas, M. C. y Funes, J. (1987). - «Application of 2thiobarbituric acid reaction to exhudates of frozen and refrigerated meats" J. Food Sci. 52, 575-79.

Turner, E. W., Payner, W. D., Montie, E. J., Bessert, M. W. Struck, G. M. y Olson, F. C. (1954). - "Use of the 2thiobarbituric acid reagent to measure rancidity in frozen pork».- Food Technol. 8, 326-330.

Vargas, A. y Gutiérrez, R. (1956). - «Ensayos sobre el enranciamiento de la manteca de cerdo. II. La prueba del ácido tiobarbitúrico».- Grasas y Aceites 7, 239-240.

Vittuci, V., Trabalza, M., Albano, M. (1986). - «Valutazione della rancidità dei grassi in prodotti di salumeria e tagli congelati».- Ind. Aliment. 25, 968-972.

Vicario Romero, I., Guillén Sans, R., Guzmán Chozas, M. (1988). - «Optimisation du dosage du TBA de l'huile d'olive 
avec extraction unique».- Rev. Fr. Corps Grass. 35, 443446.

Vicario, I., Guillén Sans, R. y Guzmán Chozas, M. (1988). - «Factores relacionados con los reactivos que pueden influenciar la determinación de la rancidez oxidativa por el ensayo del ATB". - An. Bromatol. 42, 115-125.

Vyncke, W. (1975). -«Evaluation of direct thiobarbituric extraction method for determining oxidative rancidity in mackerel (Scomber scombrus, L)».- Fette, Seifen, Anstrichm 77, 239-240.

Vycke, W. (1978). - «L'usage d'alginates pour le glaçace du hareng congelé». Rev. Agric. (Brussels) 31, 911-917.

Wada, Y. Kurangano, T., Fuse, S. y Takai, Y. (1978). - «Effects of sugaralcohol on the quality of cookies. Effects of maltitol on the oxidation of fats in cookies».- Kaseigaku Zasshi 29(8), 510-515.

Willemont, C., Fillion-Delorme, N. y Wood, D. F. (1987).«Effects of nitrite on lipid degradation during storage of cooked pork meat».- Can Inst. Food Sci. Technol. J. 20(2), 70-74.

Witas (1972). - Optimum conditions for determining 2thiobarbituric acid method using alkaline hydrolisis".- Rocz. Technol. Chem. Zywn. 22, 271-278.

Yang, G. C., Qiang, W., Morehouse, K. M., Rosenthal, I., Ku, Y. y Yurawecz, P. (1991). - «Determination of hydroperoxides in edible oils by electron spin resonance, thiobarbituric acid assay, and liquid chromatographychemiluminiescence techniques».- J. Agric. Food Chem. 39, 896-898.

Yu, T. C. y Sinnhuber, R. O. (1962). - «Removal of interfering pigments in determining malonaldehyde by the 2thiobarbituric acid reaction». - Food Technol. 16, 115-116.

Yu, T. C. y Sinnuber, R. O. (1967). - «An improve 2-thiobarbituric acid (TBA) procedure for the measurement of autoxidation in fish oils". J. Am. Oil Chem Soc. 4, 256-258.

Yun, J., Shahidi, F., Rubin, L. J. y Diosady, L. L. (1987). - «Oxidative stability and flavor acceptability of nitrite free meat-curing systems".- Can Inst. Food Sci. Technol. J. 20(4), 246-251.

Zisper, M. W. y Watts B. M. (1962). - «A modified 2-thiobarbituric acid (TBA) method for the determination of malonaldehyde in cured meats".- Food Technol. 16, 102-104.

Recibido: Octubre 1996 Aceptado: Marzo 1997 